\section{A real-time multiuser foreground, single-user background system for the PDP-9 computer}

\author{
GEORGE LAUER \\ Science Center, North American Rock well Corporation \\ Thousand Oaks, California 91360
}

We have developed a software system for a PDP.9, with fixed head disk, to allow up to 16 remote laboratories to use the central PDP-9 facility for their experimental data acquisition and control needs. Timesharing is performed on a "demand" basis, using the hardware automatic priority-interrupt option. The real-time acquisition and control aspects are separated from the data reduction in the foreground by using a batch process foreground mainstream. The background is used primarily as a systems device for program updates and communications with a CDC terminal connected to a CDC 6600 computer. The philosophy, both software and hardware, developed for this heterogeneous user environment will be discussed.

As physical research methods have become more and more sophisticated, scientists have gone more and more toward automatic data acquisition equipment. The combinations of relatively simple hardware control and programming capability have given experimentalists a large degree of flexibility. It has, however, brought on numerous problems in the research laboratory. The first of these problems is that the minicomputer becomes an end unto itself. The scientist, rather than working in his original discipline, becomes a computer scientist expert. The second problem is that the true cost of minicomputer data acquisition systems is quite high. This is true in spite of the fact that the hardware costs for the minicomputer are decreasing rapidly. In the true cost, one must include such diverse items as the cost of programming, the cost of setting up a given experiment, and the cost of maintaining the rather sophisticated equipment that has been purchased. Another major problem is that the programming efficiency of the small machines is relatively low. It would be most desirable to have the programming capabilities offered by a large machine and yet keep the flexibility of having the computer on-line.

The North American Rockwell Science Center is made up of a number of scientists, each working in a specific discipline. This means that our data acquisition needs are extremely diverse. About 2 years ago, the Science Center management decided to attempt to install a central capability which would allow each of the experimentalists to have in his lab the effective capability of a small computer and yet simultaneously have the power of a large computer available for data analysis. After a rather lengthy evaluation, it was decided to purchase a PDP-9 for this task. The configuration of the PDP-9 includes 16,000 words of memory, a memory protect option, automatic priority interrupt, and a Burroughs million-word fixed head disk.

When we first purchased the machine, we decided that it would be of great value to run in a foreground/background environment, so that we might be able to develop programs on the machine while we were simultaneously. running data acquisition programs. We, therefore, tailored our installation to the foreground/background system, as supplied by Digital Equipment Corporation. Our first impression of the system indicated to us that it was written with the idea of one foreground user and one background user in core simultaneously. At this point, it was important to differentiate between the concept of a user and the concept of a device physically connected to the machine. In our context, a user is an experimentalist in a laboratory who may have one or more devices that he has hooked up to the input/output bus of the machine. Our initial goal has been to write a supervisory program which will handle 16 users in core simultaneously. We have carefully surveyed the needs of our particular users and have found that most of their data acquisition requirements are, in reality, rather simple in terms of the amount of programming necessary to input the data and perhaps control a number of experimental variables in the laboratory. The data analyses required, however, are in many cases quite sophisticated and would require considerably more core than the data acquisition portion of the operation. We have, therefore, structured the operating system in a three-tier manner. Each user is allowed 512 words in a partition of core which is devoted solely to data acquisition. This partition is core resident throughout the length of the experiment. Access to the program is via a dedicated channel on the automatic priority-interrupt system. This guarantees each experimentalist that his data will be acquired within the time allowed. It was also found that in most cases the experimentalists considered real-time data analysis as being within the frame of their reaction time, that is, if the data comes back in 10-15 sec or, in many cases, within a minute or two, the experimentalist is quite satisfied. The second tier of our system is a queued mainstream area which allows each experimentalist to call, from within his partition, for a program to be loaded and run and then sign off, allowing the next user to go on. It is anticipated that most of these programs would run perhaps 5 to $10 \mathrm{sec}$ and then sign off. The last tier of the system is the background portion of the DEC foreground/background monitor, allowing systems' programmers and experimentalists to develop programs while the other two tiers are in operation. The details of the supervisory program will be discussed below.

\section{HARDWARE CONSIDERATIONS}

In order to implement our concept of a multiuser system, it was required that we run cables, as long as $600 \mathrm{ft}$, to the various laboratories from the central site. Figure 1 shows the general configuration of the system. The input/output bus is brought to a specially constructed interface panel. At this point, a set of receivers, W107s, and a set of drivers, B163s, are connected to the data lines. This, in effect, splits the two-way input/output bus of the PDP-9 into two one-way buses, data in and data out.

Most modern instrumentation is now being built of digital integrated circuits that operate from $+5-V$ supplies. It was decided to anticipate the use of such devices and, therefore, all the signals going to the various labs are converted at the interface panel to 0 - and 3.6-V signals, rather than the standard PDP-9 0- and 3-V signals.

To drive the very long cable lengths necessary, we have employed Texas Instruments Type SN75000 line receivers and drivers, using balanced pair cable of nominal 93-ohm impedance. At each laboratory we have a set of receivers and drivers on a party-line basis. This, in effect, gives the laboratory the input/output bus of the PDP-9. In addition, each laboratory receives the device selector 


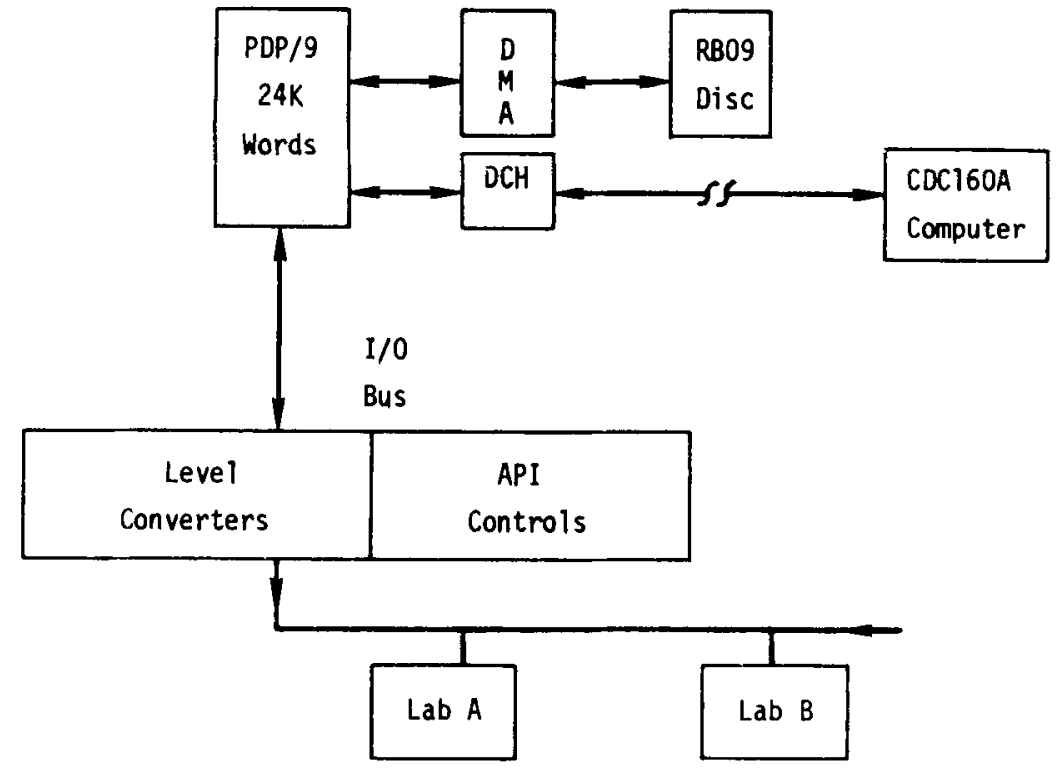

Fig. 1. Configuration of multiuser system.

lines, input/output, sync, pulse, skip-although it turns out skip is not particularly useful-and a unique interrupt line attached to the automatic priority-interrupt system. In effect, the interfacing, insofar as the users are concerned, looks very similar to the interfacing required for a PDP-8, which does not have the skip attached and, therefore, all devices must be interrupt driven. In addition, at the interface panel we have built an interprocessor controller which allows the PDP-9 to communicate with the Control Data Corporation Model 160A computer, which in turn is connected via telephone lines to a CDC 6600 computer. The controller works through the PDP-9 data channel and through the buffer channel of the $160 \mathrm{~A}$. This gives the experimentalist the capability of obtaining data in his laboratory, transferring it via the PDP-9 and the 160A, after due formatting, to the 6600 . At the 6600 the sophisticated data analyses sometimes required can be accomplished at greatest efficiency. The results are sent back, again via the $160 \mathrm{~A}$ and the PDP-9, to the experimentalist, where he can get immediate plotout of the data or, depending upon the economics of the situation, he can have a Teletype in the lab giving out the data. Effectively, our experimentalists will have a 6600 computer as a peripheral piece of equipment to their laboratory instrumentation.

Because of the long cable lengths involved, the use of IOT pulse is different from that in a standard system. IOT 1 is used to read data into the computer. Because of the propagation delay, it looks like IOT 2 by the time the data is actually read in. IOT 2 is used to switch to the user.

\section{SUPERVISORY SOFTWARE}

Supervisory software consists of a number of major subprograms. The subprograms are: (1) a special loader to load the partition-resident data acquisition programs into the user, (2) a special disk driver, (3) a communicator for foreground typewriter which can allow the operator to determine the status of the system at any time, (4) a terminator which ends the partition programs after the completion of an experimental run, and (5) a special handler to handle the queue for the mainstream time sliced programs run in the foreground. Each of these programs will be discussed below.

In order to give each experimentalist sufficient storage for his data, we have allocated 40 tracks of the RBO9 disk for the NR user system. These tracks, 160 through 199, remove Disk Unit 6 from the foreground/background system, and, therefore, all references to Unit DK6 in the .DAT table have been removed. Each user is allowed one or two tracks, depending upon his needs for storage of the data. The disk-driver program services the user requests for the data, both in the partition programs and in the mainstream programs. The communications between the PDP-9 and the $160 \mathrm{~A}$ are in the form of a driver, analogous to any of the other peripheral drivers that run in the foreground/background system. Thus, a user acquires his data in whatever format output by his particular piece of equipment. The data is stored on the user's track. When the equipment is finished, the mainstream program can convert this data into suitable IOPS formatted data for storage onto one of the regular foreground/ background disk units, and, whenever it is appropriate, the PDP-9/160A driver converts the IOPS data into the format required by the $\mathrm{CDC} 6600$ computer and transmits it to the 6600 .

\section{GENERAL SOFTWARE CONSIDERATIONS}

The NR supervisory has been designed so that it does not interfere with the DEC monitor system. It, when loaded, initializes a user map that indicates which users are in a partition at any given time. It also puts transfer vectors in the API slots of each user pointing to the loader subprogram. There are two categories of users: slow and fast. Slow users transfer data at a rate no faster than $1 / \mathrm{min}$. However, their experiments may last as long as 3 weeks. Such users share a single partition in core. When the datum is ready to be transferred to the computer from the laboratory, the lab equipment signals the computer, which, when it finds the slow partition available, loads the current program of the slow user into core, causes the data to be transferred, and then saves the program back on the disk. When a fast user is ready to begin an experiment, he signals via an interrupt to his API slot. This causes the loader to load his program into a free partition, or, if none are available, it causes the loader to checkpoint the background, load the background program onto the disk, and then, using the newly freed space, load the user from the disk into core. When the user is finished, he gives a request to the terminator program which fixes up the user map to indicate that his program is not in core anymore and, if space is available, reloads the checkpointed background.

\section{Loader \\ MAJOR SUBPROGRAMS}

The first interrupt from any user always goes to the loader, which then puts an effective holdoff into the respective API slot until the program is actually loaded. The loader determines whether any partitions are available in foreground free core and, if so, proceeds to load the user's program. If no space is available in foreground free core, the background is checkpointed and then run out onto the disk. The API slot of the requesting user is then reset to the entry of the user's program. To simplify programming considerations, all partition programs are required to look similar with respect to entry and exit. Thus, the sixth word of each partition program is the entry from the API. The first five locations are reserved to save the registers upon API entry from some other program. The loader can also be called from within a partition program in those cases where the user finds that he must chain his programs from off of the disk. The loader recognizes two 
types of programs: saved programs, which have run before and have been temporarily halted, and clean programs, which have been just taken off of the disk units of the foreground/background system. When the loader loads clean programs, it makes up a bit map determining the relocateability of each word of the user's program. This is to allow reloading of this partition program in another segment of core, if it becomes necessary. It also allows the user to request that the current program, with all of its counters and parameters set, be saved for future use to allow for chaining procedures. Users may not use global symbols within partition programs, except for those specific locations that are necessary to call the various NR supervisory segments.

\section{Terminator}

The terminator performs two functions. It can save a user's partition on Disk Unit 2 of the foreground/background system, and it can terminate a user's program at the logical conclusion of his experiment. In the former case, the saved program is placed as a named file along with the bit map on DK2 and the partition is freed for another user. If a user requests termination, the terminator resets the API slot to point to the loader and turns off the corresponding bit in the user status register, which is an auxiliary piece of hardware installed as part of the interface panel. This turns off the light in the user's laboratory, which indicates that his program is no longer in core. The terminator then checks to see if the background has been check pointed. If it has, it determines whether sufficient free space is now available, and, if space is available, it loads in the background, reinstates the appropriate registers and locations, and allows the background to run.
Disk Driver

The disk driver may be called by any of the partition programs and by any mainstream program. Its prime function is to read or write data to and from the disk, primarily Tracks 160-199. A user may request that any number of segments of 64 words each be transferred to any portion of his available disk space in any format whatsoever. Thus, the user has a random-access mass storage capability available to him. It is up to the user to ensure that he does not write over or access any other user's disk area. The disk driver on entry checks to see if the background/ foreground system is using the disk. If it is, the driver resets the API slot to point to itself and saves the appropriate registers. It then debreaks until the disk has completed its current operation. At that time it transfers the data to or from the disk from the user's partition and, on completion, returns control back to the foreground/background system disk driver. The disk driver is capable of handling a queue of requesting users of length 16 . When a disk transfer operation is completed, control may revert to the user program that has requested such transfer, depending upon the type of call made to the NR disk driver. This is to allow users to set up a double buffering scheme for transfer of data obtained at rates higher thar $500 \mathrm{~Hz}$.

\section{Mainstream Handler}

This program handles a queue for waiting mainstream job requests and loads them in at the proper time. Mainstream programs may be no longer than 4,000 words. It is anticipated that these jobs will require about 5-10 sec to complete. They include such various tasks as performing fast Fourier transform, reformatting of data, and displaying of data in the laboratory which has been obtained during a prior data acquisition run.

\section{SUMMARY}

The system that has been described is definitely not optimum. It is expected that is will grow as more and more users go on-line and as we gain experience in the operation of a multiuser system. It is clear, however, that any attempt at this concept must clearly differentiate between the human user and the hardware device. The DEC foreground/background system, as delivered, is clearly written to handle devices, and definitely not users. It would be far superior to have a system where the differentiation is made. Hardware devices require software drivers which, once fully debugged, need never be changed again. The hardware devices are linited by the electronics that have been wired in. On the other hand, the only justification to a user for going on-line to a computer is the flexivility allorded by the computer. Thus, any software system must allow the user to change his program easily and rapidly. so that he may work interactively ani not have to consider the various interactions of his program with the overall system. This rules out writing standard hardware-type drivers to drive the software of the user. It is anticipated that eventually the complete foreground system, as written by Digital Equipment, will be replaced by a system more amenable to a multiuser environment. However, the writing of such a set of programs will have to be deferred until time and funds become available. 